THE KURUME MEDICAL JOURNAL

Vol. 28, p. 159-164, 1981

\title{
Influenza Vaccination in Hemodialysis Patients
}

\author{
MASARO KAJI, KENJI TAKAO, HIROSHI MINAMI, HIDEYO NATORI, \\ KENJI SAEKI, YASUMI SATO, KENJI KOBAYASHI, KOZABU ISHII, \\ YOSHIRO KUROSAWA, HIDEHARU UMENO, YUJI HIROMATSU \\ AND SEIZABURO KASHIWAGI*
}

Department of Internal Medicine, Kurume University School of Medicine, Kurume, 830 and
${ }^{*}$ Department of Internal Medicine, Kyushu University School of Medicine, Fukuoka, 812 Japan

Received for publication April 25, 1981

\begin{abstract}
Summary: Sixty-one long-term hemodialysis patients were inoculated with influenza vaccine. Antibody responses measured by hemagglutination inhibition test were comparable to that of controls. Side reactions of vaccination were similar to controls in frequency and severity. No adverse effects were observed in the clinical course of inoculated hemodialysis patients.

Influenza vaccination can be recommended for annual immunization of hemodialysis patients as a group at high risk from influenza infection.
\end{abstract}

Key words: Influenza - Influenza vaccine - Immunization - Vaccine adverse reaction - Hemodialysis

\section{Introduction}

The recent increase in hemodialysis patients in Japan has highlighted the importance of influenza prevention to such patients as they are considered to be at high risk from influenza. Hemodialysis patients were evaluated following influenza vaccination for antibody response, possible adverse effects on renal function and other side effects. This report describes the results obtained.

\section{Subjects and Methods}

Subjects: Sixty-one patients (male 35, female 26) were examined. Their ages ranged from 23 to 74 years (mean 47.5) and terms of hemodialysis ranged from 10 days to 83 months (mean 24.5 months). Diseases which caused chronic renal failure are listed in Table 1. None of the patients were treated with corticosteroid or im- munosuppresive drugs during the study. Fifty-eight adults, either healthy or with minor illnesses, served as controls.

Vaccine: Influenza HA vaccine supplied from the Study Committee of Influenza Vaccine (Director Dr. H. Fukumi) contained A/USSR $/ 92 / 77$ (H1N1) $400 \mathrm{CCA} / \mathrm{ml}$ eq. and $\mathrm{B} /$ Kanagawa $/ 3 / 76300 \mathrm{CCA} / \mathrm{ml}$ eq.

Study design (Fig. 1): In the study group, $0.3 \mathrm{ml}$ of vaccine was inoculated subcutaneously three times at 2 week intervals. Thus the total amount of vaccine inoculum per patient was $0.9 \mathrm{ml}$. In the control group, two doses of $0.5 \mathrm{ml}$ (total $1.0 \mathrm{ml}$ ) were given 2 weeks apart as indicated for general use. Blood samples were collected from the study group four times: before the first inoculation and every 2 weeks until 2 weeks after the third inoculation. In the control group, blood samples were taken before the first inoculation and 4 weeks after the second inocula- 
TABLE 1

Underlying disease in hemodialysis patient

\begin{tabular}{l|c}
\hline \multicolumn{1}{c|}{ Disease } & No. of Patient \\
\hline Chronic glomerulonephritis & 53 \\
Diabetic nephropathy & 5 \\
Systemic lupus erythematosus & 1 \\
Malignant hypertension & 1 \\
Gout & 1 \\
\hline \multicolumn{1}{c|}{ Total } & 61 \\
\hline
\end{tabular}

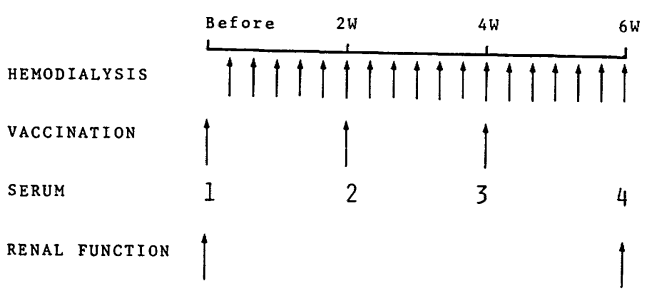

Fig. 1. Study design tion. Serum antibody against two vaccine strains was measured by hemagglutination inhibition (HI) test.

Blood urea nitrogen (BUN), creatinine and hemoglobin were also examined in the study group in the initial and final blood samples.

The study was carried out between December 1978 and March 1979.

\section{Results}

Antibody responses: Distributions of $\mathrm{HI}$ titer before the first inoculation, and 2 , 4 , and 6 weeks after the first inoculation are shown in Figs. 2 and 3. For two strains, A/USSR/92/77 and B/Kanagawa/ $3 / 76$, good response was observed from 2 to 6 weeks after the vaccination with slight change.

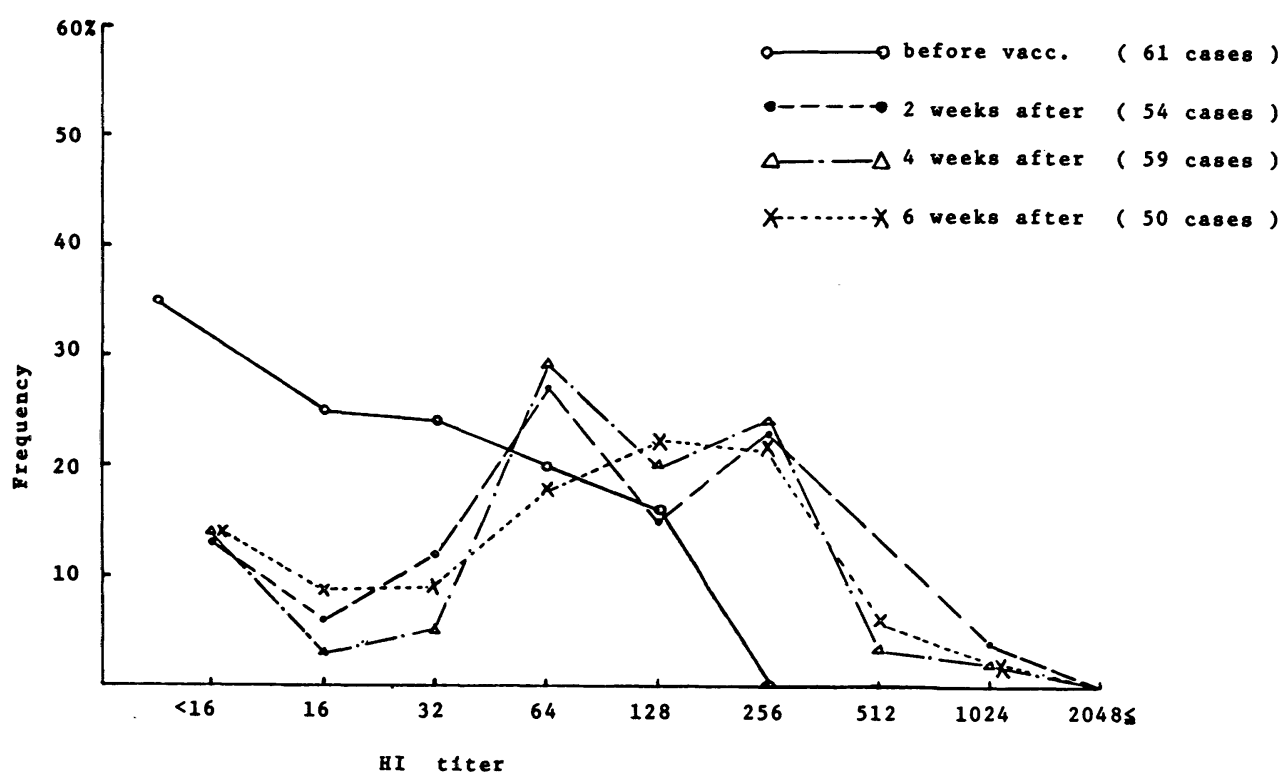

Fig. 2. Distribution of $\mathrm{HI}$ titer against A/USSR/92/77 before and after influenza vaaccination. 


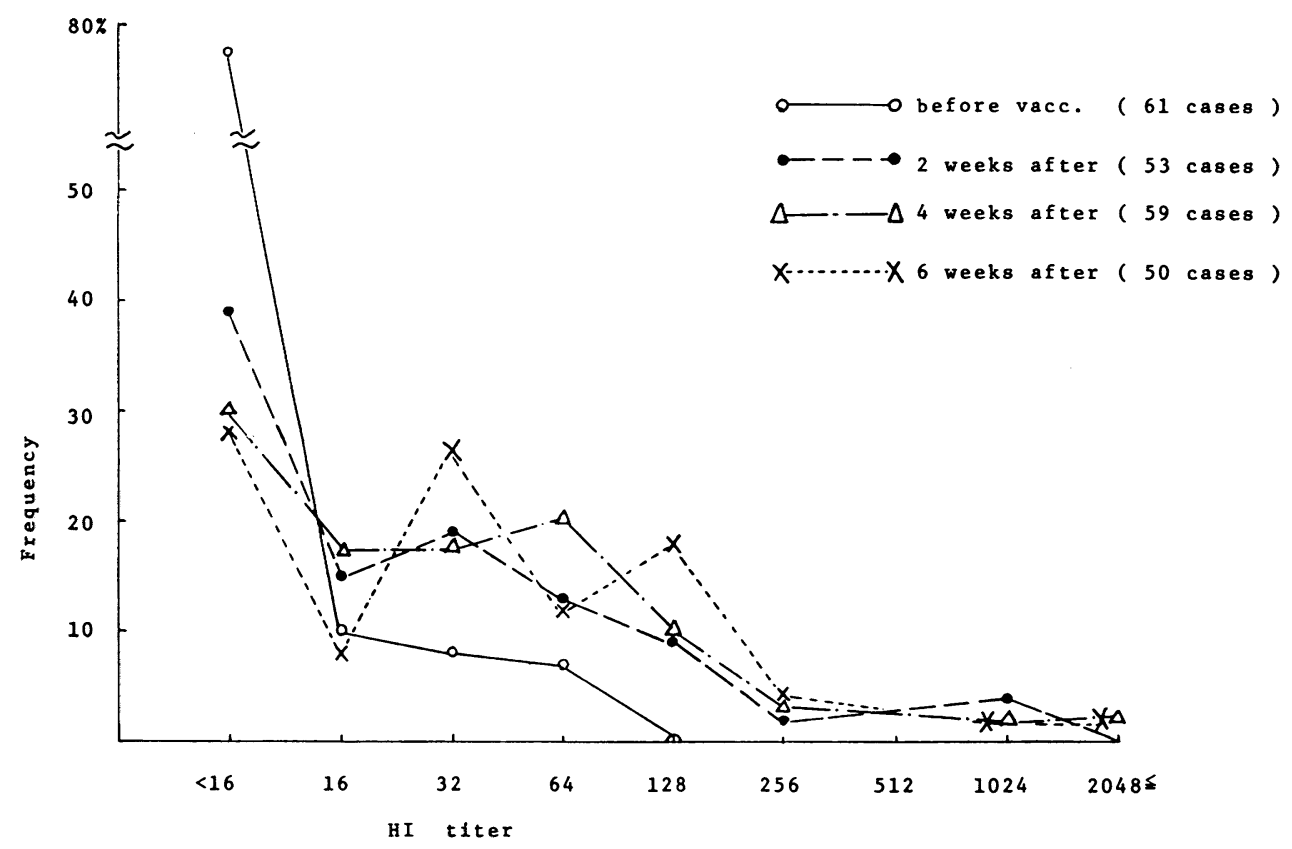

Fig. 3. Distribution of $\mathrm{HI}$ titer against B/Kanagawa/3/76 before and after influenza vaccination.

A/USSR/92/77

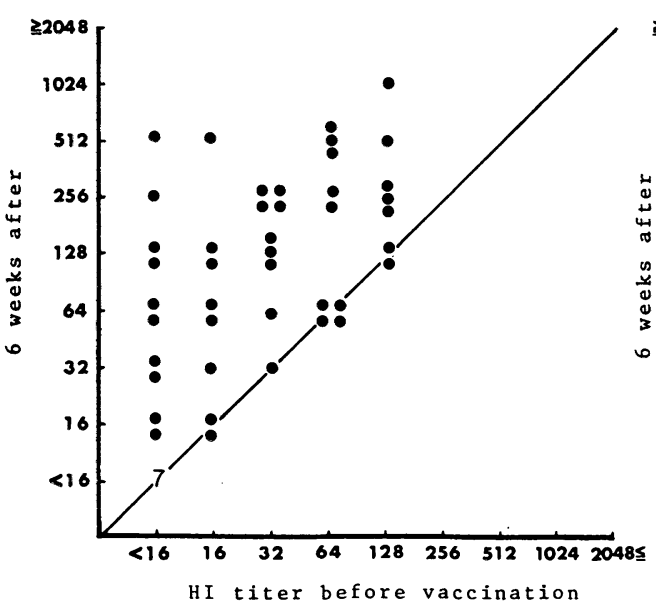

B/Kanagawa/3/76

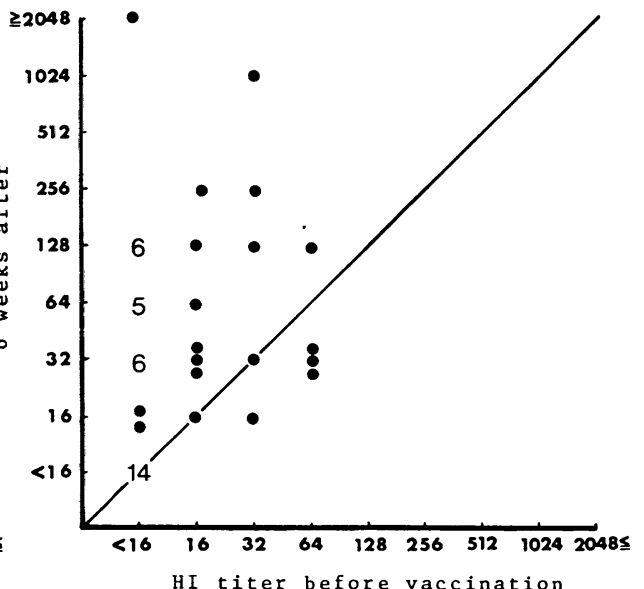

Fig. 4. Antibody response in 50 hemodialysis patients before and 6 weeks after influenza vaccination. 
Seroconversion rate (4-fold or greater rise in HI titer) was $50 \%$ for A/USSR/ $92 / 77$ and $48 \%$ for B/Kanagawa/3/76 respectively (Fig. 4). Table 2 shows seroconversion among 61 patients with varying underlying diseases. In the control group, a 4-fold or greater rise in HI titer was observed in 31 (53\%, for A/USSR/92/77) and $21(36 \%$, for $\mathrm{B} /$ Kanagawa/3/76) out of 58 subjects.

Adverse reactions: As shown in Table 3 , local and systemic reactions due to vaccination were observed in few cases. The frequency and severity of the reactions were similar to those in the control group. The incidence and severity also were comparable to those found in another population of approximately 600 cases inoculated with the same vaccine. No serious adverse reactions were observed in either hemodialysis patients or the control group throughout the study.

Renal function: BUN, creatinine, BUN/ $\mathrm{Cr}$ and hemoglobin examined in the initial and final blood samples showed no significant changes (Fig. 5). Thus, influenza vaccination seems to have no influence on the course of hemodialysis patients.

TABLE 2

Seroconversion in 61 hemodialysis patients by underlying disease

\begin{tabular}{l|c|c|c}
\hline \multirow{2}{*}{ Disease } & No. of & \multicolumn{2}{|c}{ No. of seroconverted cases for } \\
\cline { 3 - 4 } & cases & A/USSR/92/77 & B/Kanagawa/3/76 \\
\hline Chronic glomerulonephritis & 53 & 27 & 23 \\
Diabetic nephropathy & 5 & 3 & 3 \\
Others* & 3 & 3 & 3 \\
\hline
\end{tabular}

* SLE 1, Malignant hypertension 1, Gout 1

TABLE 3

Adverse reactions of influenza vaccine in 61 hemodialysis patients

\begin{tabular}{|c|c|c|c|c|}
\hline & \multirow{2}{*}{ Reaction } & \multicolumn{3}{|c|}{ Influenza vaccine } \\
\hline & & $1 \mathrm{st}$ & $2 \mathrm{nd}$ & $3 \mathrm{rd}$ \\
\hline \multirow{4}{*}{ Local } & Redness & - & - & - \\
\hline & Pain & - & - & - \\
\hline & Induration & 1 & - & - \\
\hline & Itching & 1 & - & 2 \\
\hline \multirow{6}{*}{ Systemic } & Chill & - & - & 1 \\
\hline & Headache & - & $1^{*}$ & - \\
\hline & Uncomfortable feeling & - & 1 & - \\
\hline & Coryza-like symptoms & - & 3 & - \\
\hline & Diarrhea & 1 & 1 & - \\
\hline & Oppression of chest & $1^{*}$ & - & - \\
\hline
\end{tabular}

\footnotetext{
* Possibly due to hemodialysis
} 

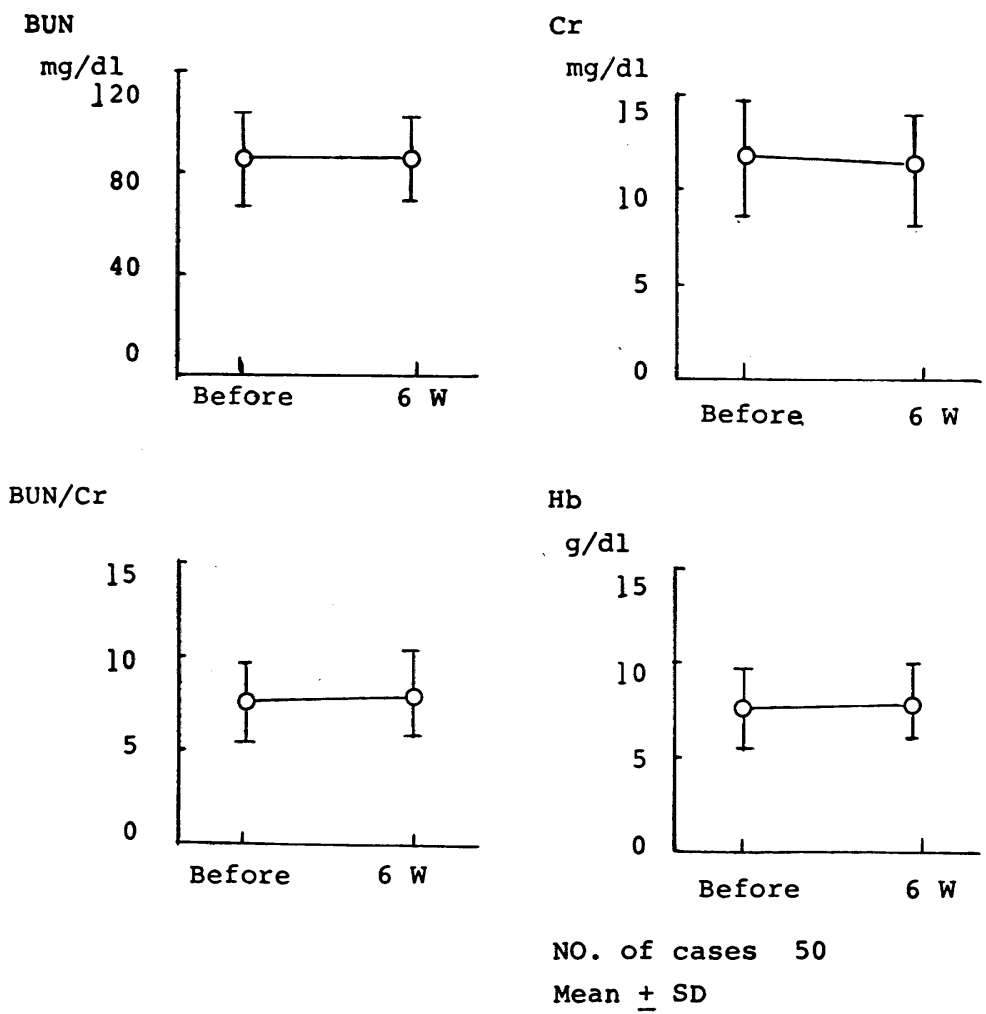

Fig. 5. Renal function and $\mathrm{Hb}$. before and after influenza vaccination.

\section{Discussion}

Impaired immunity in chronic renal failure patients has been proven (Goldblum and Reed, 1980) and interest now focuses on the care of hemodialysis patients and renal transplant patients (Carroll et al, 1974; Kumar et al., 1978; Briggs et al., 1980). Because hemodialysis patients are at high resk from influenza, the need for evaluation of influenza vaccine is reasonable.

Although depressed cell-mediated immunity and variably depressed humoral immunity have been shown in patients on hemodialysis, it has also been shown that the antibody responses to influenza vaccine in hemodialysis patients is equal to that of healthy controls.
Jordan et al. (1973) reported that 22 hemodialysis patients exhibited comparable antibody response to 28 normal control subjects after inoculation with inactivated whole particle bivalent vaccine. Haldiman and Descoedres (1978) described significant rises in antibody titer after inoculation with subunit or adjuvant containing whole virus vaccine in 50 hemodialysis patients. Kashima et al. (1978) also reported similar antibody responses in 18 hemodialysis patients and healthy controls.

Before suggesting annual influenza immunization for hemodialysis patients, more data are needed on the antibody response, and possible adverse effects on renal function, as well as other side effects.

In our study, as a special precaution 
hemodialysis patients received one dose of $0.3 \mathrm{ml}$ instead of $0.5 \mathrm{ml}$ of vaccine as indicated. These patients then received 2 additional doses of $0.3 \mathrm{ml}$. Thus, hemodialysis patients were inoculated with a total of $0.9 \mathrm{ml}$ of vaccine compared to the control group which received $1.0 \mathrm{ml}$ vaccine in 2 divided doses.

The results obtained in our study on 61 patients showed seroconversion rates and rises in $\mathrm{HI}$ titer for $\mathrm{A}$ and $\mathrm{B}$ vaccine strains comparable to those of the control. However, slight differences in vaccination schedule and serum collection must be considered.

In the winter of 1978-1979, little influenza activity was reported in our district and no influenza was observed clinically among the subjects under study. Thus, it can be assumed that antibody responses in the study group were due to vaccine inoculated.

Adverse reactions (local or systemic) to influenza vaccine in the 61 hemodialysis patients were not significantly different in frequency or severity compared to the controls. Furthermore, examination of renal function before and after vaccination produced no remarkable adverse effects on the clinical course of the patients.

Further studies should include detailed observations of antibody response in relation to the various underlying diseases such as chronic glomerulonephritis and SLE which result in chronic renal failure.

These results suggest influenza $\mathrm{HA}$ vaccine is well tolerated by hemodialysis patients and protective effects can be expected.

\section{References}

Briggs, W. A., Rozek, R. J., Migdal, S. D., Shillis, J. L., Brackett, R. G., Brandon, F. B., Mahajan, S. K. and McDonald, F.D. (1980). Influenza vaccination in kidney transplant recipients: cellular and humoral immune responses. Ann. Int. Med. 92, 471-477.

Carroll, R. N.P., Marsh, S.D., O'Donoghue, E. P. N., Breeze, D.C. and Shackman, R. (1974). Response to influenza vaccine by renal transplant patients. Brit. M. J. 2,701-703.

Goldblum, S. E. and REED, W.P. (1980). Host defenses and immunologic alterations associated with chronic hemodialysis. Ann. Int. Med. 93, 597-613.

HaldimanN, B. und Discoeudres, C. (1978). Grippeimpfung bei Dialysepatienten. Schweiz. Med. Wschr. 107, 52-55.

Jordan, M.C., Rousseau, M.D., Tegtmeier, G.E., Noble, G. R., Muth, R. G. and Chin, T.D. Y. (1973). Immunogenicity of inactivated influenza virus vaccine in chronic renal failure. Ann. Int. Med. 79, 790-794.

Kashima, T., Shimada, T., Irie, Y., Odaka, M., Nomura, Y., Soma, M. and OKaWA, M. (1978). On the antibody responses and side reactions of influenza vaccination in hemodialysis patients. J. Jap. Soc. Dialsysis Therapy, 11, 473-474 (in Japanese).

Kumar, S. S., Ventura, A. K. and B. VanderWERF. (1978). Influenza vaccination in renal transplant recipients. J.A.M.A. 239, 840842. 